\title{
AORTIC FUNCTION IN PATIENTS DURING INTRA-AORTIC BALLOON PUMPING DETERMINED BY THE PRESSURE-DIAMETER RELATION
}

Christodoulos Stefanadis, MD, FESC, FACC

John Dernellis, MD

Eleftherios Tsiamis, MD

Costas Stratos, MD

Ioannis Kallikazaros, MD

Pavlos Toutouzas, MD, FESC, FACC
Objective: The physiologic basis for the hemodynamic and clinical improvement achieved by the use of intra-aortic balloon pumping in patients with cardiogenic shock has not been clarified in all its aspects. This study evaluated the possible contribution of pump-induced alterations of aortic mechanics to the overall benefit gained by the implementation of this therapeutic modality in patients with acute heart failure of ischemic origin. Methods: The aortic pressure-diameter relation was obtained by use of an intravascular catheter for aortic diameter measurements developed in our institution and previously validated, simultaneously with a catheter-tip micromanometer for aortic pressure measurements at the same aortic level. Aortic function indices were compared before and during intra-aortic balloon pumping in 12 patients with cardiogenic shock. Results: Intra-aortic balloon pumping increased cardiac index and aortic distensibility by $24 \%$ and $30 \%$, respectively, and reduced myocardial oxygen demand by $31 \%(P<.001$ for all alterations). Energy loss caused by aortic wall viscosity increased by $207 \%(P<.001)$. The aortic diameter augmentation index increased by $68 \%(P<.001)$; the aortic pressure augmentation index decreased by $117 \%(P<.001)$. Linear regression analysis showed that cardiac index and myocardial oxygen demand were related with the aortic stiffness constant both before and during intra-aortic balloon pumping. Conclusions: During intra-aortic balloon pumping, aortic distensibility was improved, and wave reflection from the arterial periphery was reduced. The relationship between cardiac index and myocardial oxygen demand and aortic stiffness suggests that improvement of the elastic properties of the aorta was an important mechanism by which intraaortic balloon pumping improved circulatory function. (J Thorac Cardiovasc Surg 1998;116:1052-9)
$\mathrm{D}$ espite the recent development of new devices capable of percutaneous insertion and near-total cardiopulmonary support outside the operating room, the intra-aortic balloon pump (IABP) remains the most commonly used mechanical cardiac support device. ${ }^{1-3}$ This device supports the left ventricle by introducing external energy into the vascular system and by reduc-

From the Hippokration Hospital, Department of Cardiology, University of Athens, Greece.

Supported in part by a grant from the Helenic Heart Foundation.

Received for publication May 8, 1998; accepted for publication July 28,1998

Address for reprints: Christodoulos Stefanadis, MD, FESC, FACC, 9 Tepeleniou Str, 15452 Paleo Psychico, Athens, Greece.

Copyright $@ 1998$ by Mosby, Inc.

$0022-5223 / 98 \$ 5.00+0 \quad \mathbf{1 2 / 1 / 9 3 4 7 5}$ ing the left ventricular afterload and, consequently, myocardial oxygen demand. ${ }^{4,5}$

Previous studies that used different techniques have shown that systemic vascular resistance, ${ }^{6}$ afterload, and myocardial oxygen demand ${ }^{7}$ are reduced during IABP. However, they do not provide information regarding possible alterations of the aortic function during IABP, despite the fact that the aorta accommodates the pulsating balloon. This kind of information may be provided precisely by the aortic pressure-diameter relation. Recently, we described a new method to obtain aortic pressure-diameter relation in conscious human beings. ${ }^{8-10}$ According to this method, aortic diameters are acquired with use of a high-fidelity intravascular catheter that was developed in our institution and that incorporates an ultrasonic displacement meter at its distal end. Aortic pressures are acquired simultaneously 
with a catheter-tip micromanometer at the same level of the vessel.

The objective of this study was to use this method to test the hypothesis that IABP improves aortic function by acutely improving aortic wall properties and dilating peripheral vasculature.

\section{Methods}

Patients. The study group consisted of 12 patients in the intensive care unit with acute heart failure caused by acute myocardial infarction or unstable angina. Patients with peripheral vascular disease, significant aortic insufficiency, aortic dissection, and bleeding diathesis were excluded. IABP was initiated in the intensive care unit in all patents either because of a lack of response to the proper administration of fluids and/or pharmacologic therapy or because of acute heart failure accompanied by refractory ischemia, in preparation for cardiac catheterization, coronary arteriography, and definitive intervention. Studies were performed after the initiation of IABP only in patients in sinus rhythm who were hemodynamically stable enough to tolerate a brief period of graded weaning of IABP support. The protocol was approved by the Ethics Committee of our institution, and all patients and/or their families gave informed consent before participating.

Measurement of aortic diameter and pressure. Instantaneous aortic diameters and pressures were recorded simultaneously and at the same point of the aorta. This technique has been recently described in detail. ${ }^{8-10}$ Aortic diameter was measured in the proximal descending aorta, which was $1 \mathrm{~cm}$ from the left subclavian artery, by a Y-shaped intravascular catheter that was developed in our institution and uses sonometry for the measurement of diameter. At each arm of the catheter, a piezoelectric crystal $(5 \mathrm{MHz}$ in frequency, $1 \mathrm{~mm}$ in diameter; Crystal Biotech, Inc, Northborough, Mass) is attached. The technical characteristics of the device include (1) resolution for assessment of changes in diameter of $10 \mu \mathrm{m}$, (2) flat $( \pm 5 \%)$ frequency response in testing up to $40 \mathrm{~Hz}$, (3) no measurable phase lag between forced oscillations of the device and the signal in the frequency response range, and (4) minimal loading on the aortic wall $(0.45 \mathrm{~g}$ per arm when the distance between the arms is $1 \mathrm{~cm})$.

Aortic pressures were recorded with a catheter-tip micromanometer (SPC-330; Millar Instruments, Inc, Houston, Tex). The transducer was calibrated electronically against mercury at the beginning of each study.

Study protocol. Patients with acute heart failure who were found to have a cardiac index greater than $1.8 \mathrm{~L} \cdot \mathrm{min}^{-1} \cdot \mathrm{m}^{-2}$ were transferred to the cardiac catheterization laboratory. All patients had dual-lumen intra-aortic balloon catheters with 40-mL balloon volumes (9F; Arrow International, Inc, Reading, Pa) inserted percutaneously via the right femoral artery. The distal tip of the balloon catheter was positioned in the descending thoracic aorta within $2 \mathrm{~cm}$ from the left subclavian artery under fluoroscopic guidance. A thermodilution pulmonary artery catheter had been inserted at an earlier

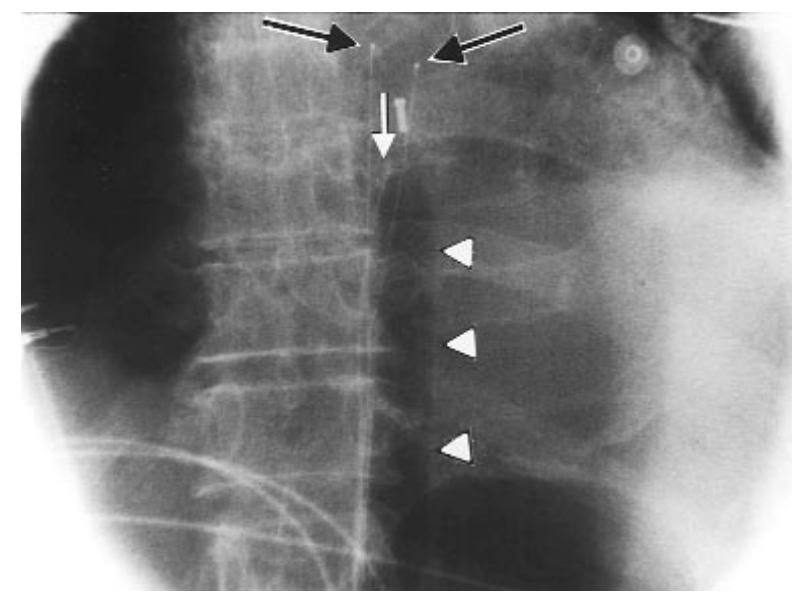

Fig 1. Fluoroscopic image shows the diameter catheter (the ultrasonic crystal-bearing tips of its Y-shaped end, black arrows), the Millar micromanometer (white arrows), and the inflated intra-aortic balloon (arrowheads), within the descending thoracic aorta.

stage of shock management. All patients were receiving an intravenous continuous infusion of heparin to maintain activated clotting time of more than 300 seconds.

For insertion of the diameter device, a long $(50-\mathrm{cm}) 8 \mathrm{~F}$ guiding sheath was introduced through a $9 \mathrm{~F}$ introducer placed in the femoral artery and positioned to the level of the proximal descending aorta within $1 \mathrm{~cm}$ from the left subclavian artery under fluoroscopic observation. The catheter (with the wires collapsed) was then advanced into the guiding sheath. Once the catheter tip was in position, the guiding sheath was withdrawn to expose completely the Y-shaped end of the catheter, which allowed the arms to spread apart until they touched the aortic wall and followed freely its movement throughout the cardiac cycle. The catheter position was frequently checked by fluoroscopy during the study to document its stability.

The catheter-tip micromanometer $(3 \mathrm{~F})$ was inserted through a $5 \mathrm{~F}$ introductory sheath punctured into the left femoral artery and was advanced to a point located minimally below the level of the pair of ultrasonic crystals (Fig 1).

Pressure and diameter measurements were obtained at an IABP/R-wave trigger ratio of $1: 1$. Then IABP was programmed serially to R-wave trigger ratios of 1:2, 1:4, and 0:1 (IABP off), and the patient's hemodynamic condition was allowed to stabilize for 2 minutes before baseline measurements were made at IABP off. No changes in patient position and intravenous fluid or drug administration were made during the periods of study. Thereafter, IABP was started again. To eliminate possible beat-to-beat variability of pressure and diameter data as the result of possible displacement of the catheters during IABP, a sampling period that consisted of 10 consecutive cardiac cycles was accepted for further analyses if the variation coefficients (standard deviation/mean) of maximal and minimal aortic pressure and diameter of that period were less than $1 \%$. 

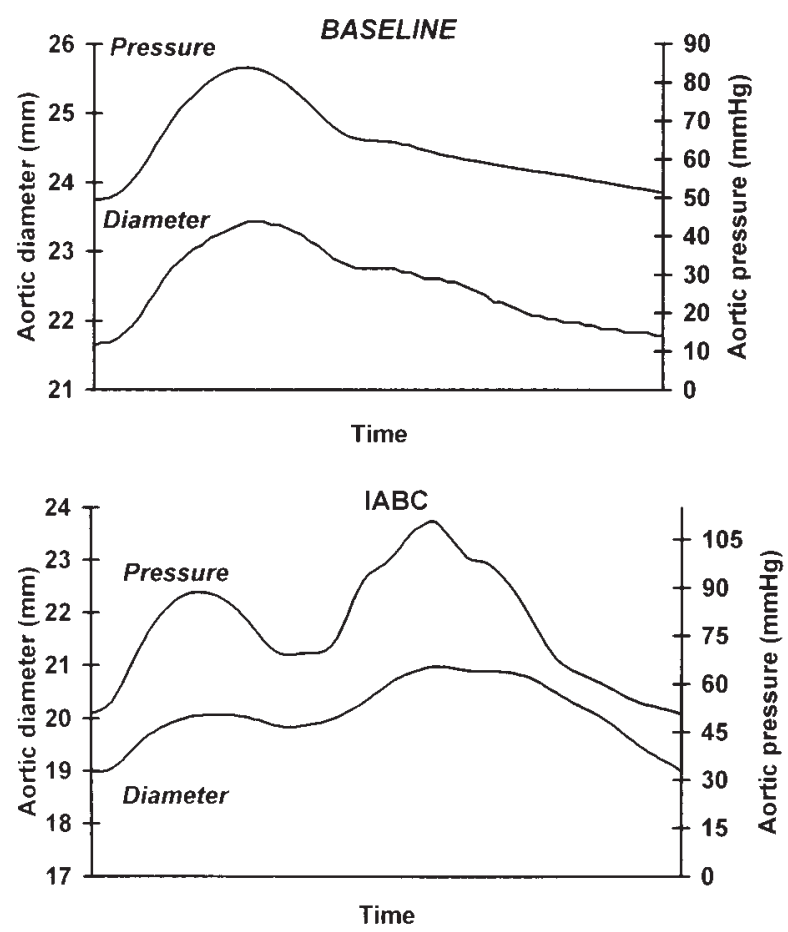

Fig 2. Aortic diameter and simultaneous aortic pressure tracings at baseline (top) and during IABP (bottom). Both pressure and diameter tracings showed 2 prominent peaks during IABP; the first occurred during left ventricular systole and the second occurred during balloon inflation.

Data acquisition. Throughout the study, electrocardiographic, aortic diameter, and aortic pressure signals were collected with a VF-1 mainframe computer (Crystal Biotech, Inc, Northborough, Mass) fitted with appropriate modules for acquisition of data and were simultaneously displayed in real time mode on a personal computer (Pentium 100; Intel Corporation, Janesville, Wis) with a multichannel 12-bit analog-to-digital converter (Data Translation Inc, Marlboro, Mass) and commercially available data acquisition software (Dataflow; Crystal Biotech). The digitized data were stored and later processed with commercially available software (Microsoft Excel for Windows; Microsoft Corporation, Redmond, Wash; Fig 2). Signals were digitized every $5 \mathrm{~ms}$. For aortic pressure and diameter values and subsequent calculations of derivative parameters, the analyses were performed on 10 consecutive cycles, and the results were averaged.

Indices of aortic elastic properties. Aortic distensibility. Cross-sectional distensibility of the aorta was calculated with the following formula ${ }^{11-14}$ : aortic distensibility $=2 \times(\max -$ $\min$ aortic diameter $) /([\min$ aortic diameter $] \times[\max -\min$ aortic pressure]), where max and min aortic parameters refer to systolic and diastolic aortic parameters at baseline when the IABP was turned off. During IABP, 2 different measurements of distensibility were calculated: the first referred to the first systolic peak of the aortic pressure and diameter trac-
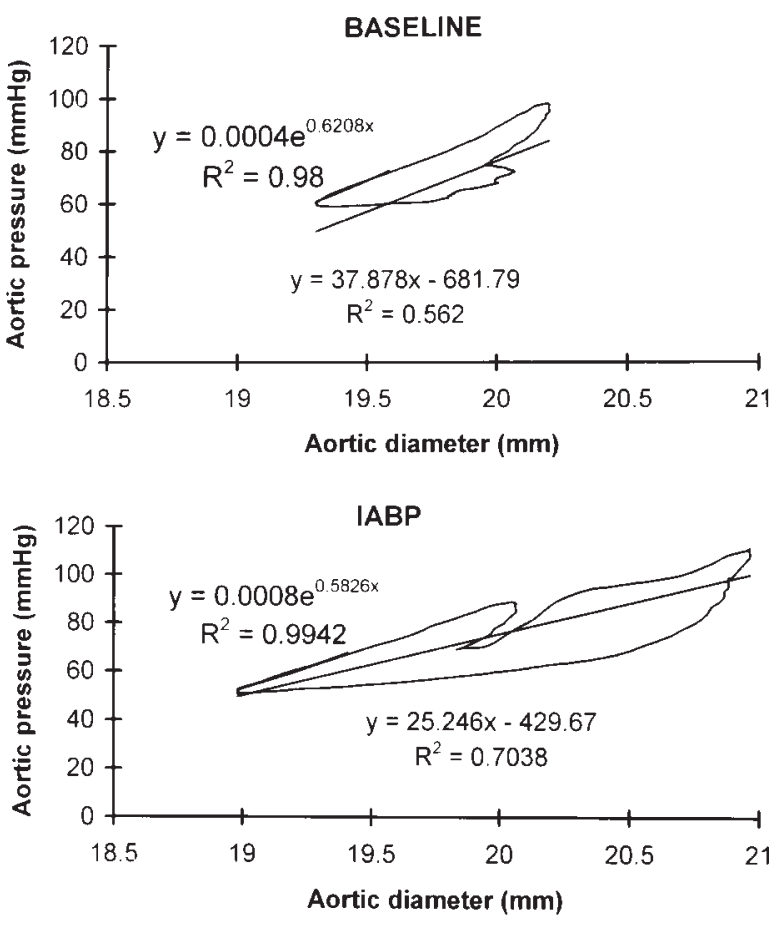

Fig 3. Clockwise aortic pressure-diameter loops at baseline and during IABP at an IABP/R-wave trigger ratio of 1:1. $\mathrm{IABP}$ resulted in changes in the contour of the pressure-diameter relationship. The loop at baseline has a steeper slope that indicates reduced elastic properties compared with the loop during IABP. The exponential regression for the calculation of the aortic stiffness constant is also shown (see text for details).

ings occurring during left ventricular systole (partial distensibility) and the second referred to the entire cardiac cycle including both left ventricular systole and balloon inflation (total distensibility).

Aortic pressure-diameter relation. Aortic pressure-diameter relation ${ }^{8-10}$ was obtained by plotting the pressure and diameter digitized data by means of a commercially available computer software (Microsoft Excel for Windows; Microsoft Corporation; Fig 3). To characterize the pressure-diameter relation and determine the aortic loop orientation, the slope and the intercept of the linear regression line of pressure versus diameter were calculated.

Aortic stiffness constant. Aortic pressure-diameter data ${ }^{15}$ obtained during left ventricular ejection, which corresponds to the ascending limb of the loop, were used for the calculation of the aortic stiffness constant. The rate of aortic pressure changes $(\mathrm{dP} / \mathrm{dt})$ was instantaneously calculated and simultaneously recorded with the high-fidelity pressure. Pressure and diameter data during the ascending limb of the loop, starting at the time when the $\mathrm{dP} / \mathrm{dt}$ curve reached zero baseline (at the beginning of the ascending limb of the loop) and ending at peak of $+\mathrm{dP} / \mathrm{dt}$, were fitted to the exponential function: $\mathrm{P}=\mathrm{b} \times \mathrm{e}^{\mathrm{a} . \mathrm{D}}$, where $P$ is the instantaneous aortic pressure 
Table I. Clinical characteristics of patients treated with IABP

\begin{tabular}{lc}
\hline Characteristics & $I A B P(n=12)$ \\
\hline Age (yr) & $55 \pm 3$ \\
Male/female (No.) & $10 / 2$ \\
Remote myocardial infarction (No.) & 5 \\
History of CABG (No.) & 2 \\
Acute myocardial infarction (No.) & 11 \\
Thrombolysis (No.) & 10 \\
Unstable angina (No.) & 1 \\
Time of study after the initiation of IABP (h) & $13 \pm 5$ \\
Coronary artery stenoses (No.) & \\
Left main & 5 \\
LAD & 12 \\
LCx & 5 \\
RCA & 3 \\
Multivessel disease (No.) & 12 \\
Vein grafts disease (No.) & 2 \\
Rescue PTCA (No.) & 10 \\
Rescue CABG (No.) & 2 \\
\hline
\end{tabular}

$C A B G$, Coronary artery bypass grafting; $L A D$, left anterior descending coronary artery; $L C x$, left circumflex coronary artery; $R C A$, right coronary artery; $P T C A$, percutaneous transluminal coronary angioplasty.

and $D$ is the instantaneous aortic diameter. The least-squares method was used for calculation of $a$ and $b$, where $a$ is the elastic aortic stiffness constant (millimeter ${ }^{-1}$ ), which determines the slope of the exponential curve, and $b$ is the elastic constant (millimeters of mercury; Fig 3).

Aortic energy loss. Energy loss as the result of the viscosity of the aortic wall was represented by the area (millimeters - millimeters of mercury) within the aortic loop.

Wave reflection indices. Wave reflections were evaluated by measuring the pressure augmentation index, ${ }^{16,17}$ defined as the ratio: pressure from inflection point to late systolic peak/pulse pressure. Beginning of pressure wave upstroke, inflection point and late systolic peak were defined by the use of the fourth derivative of pressure.${ }^{17}$ Furthermore, reflection waves were evaluated by measuring the diameter augmentation index, defined similarly as the ratio: diameter from inflection point to late systolic peak/pulse diameter (Fig 4).

Myocardial oxygen consumption. Myocardial oxygen consumption was estimated by the calculation of the tension-time index (TTI). TTI was obtained from the measurement of the area under the systolic portion of the aortic pressure curve. ${ }^{6,18}$

Statistical analysis. The Student paired $t$ test for repeated measurements was used for comparisons between continuous variables with and without IABP support. Corrections were evaluated with the Pearson's coefficient of correlation.

\section{Results}

Clinical characteristics. The baseline characteristics of patients treated with IABP are shown in Table I. All patients who were hemodynamically stable enough to
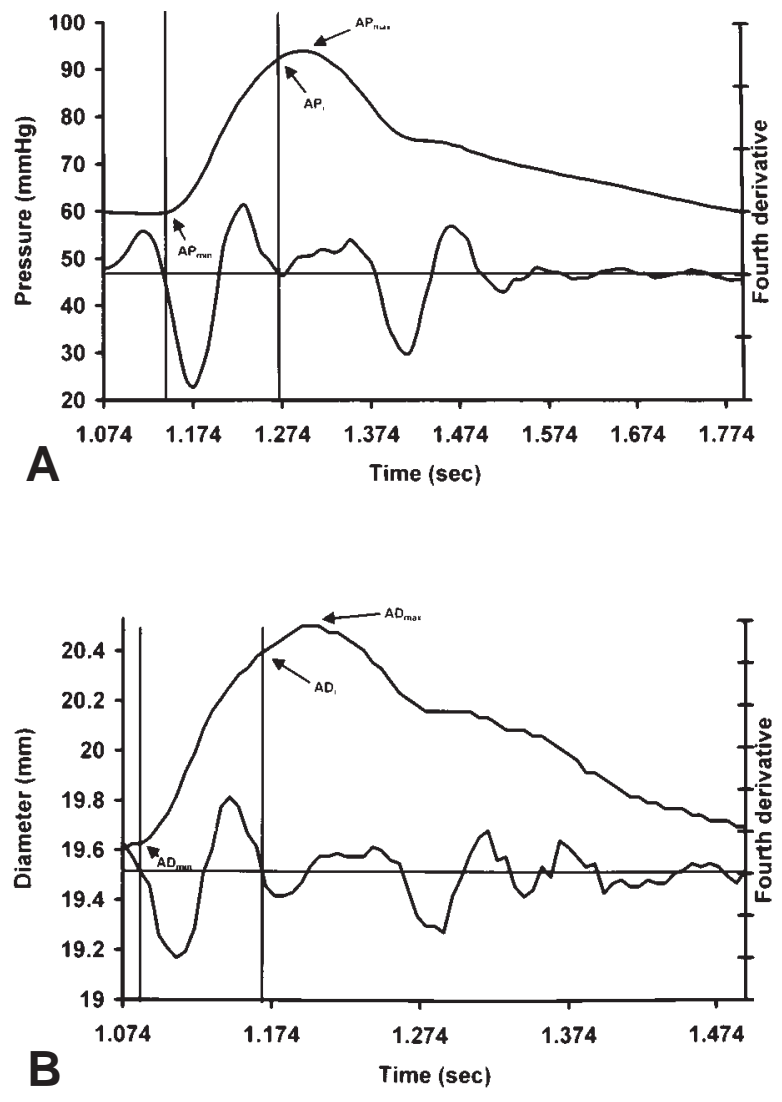

Fig 4. Graphic plotting of the fourth derivative of aortic pressure $(A P)$ and diameter $(A D)$. A, First zero crossing of the fourth derivative corresponds to minimal aortic pressure and diameter $\left(\mathrm{AP}_{\text {min }}\right.$ and $\mathrm{AD}_{\text {min }}$, respectively). B, Second zero crossing in same direction (from above to below) corresponds to the inflection point $\left(\mathrm{AP}_{\mathrm{i}}\right.$ and $\mathrm{AD}_{\mathrm{i}}$, respectively). The maximal aortic pressure and diameter $\left(\mathrm{AP}_{\max }\right.$ and $\mathrm{AD}_{\text {max }}$, respectively) correspond to the peak of pressure and diameter waveforms.

tolerate a brief period of weaning of IABP support were studied before rescue intervention. Ten patients underwent successful rescue angioplasty (7 angioplasties in the left anterior descending coronary artery, 1 angioplasty in the left circumflex, 1 angioplasty in the right coronary artery, and 1 angioplasty in the vein graft). The remaining 2 patients underwent rescue coronary artery bypass grafting. All patients improved clinically and were successfully weaned from IABP. IABP was discontinued within 24 hours in 1 patient because of hemorrhagic complications at the femoral artery puncture site after the study had been completed.

Hemodynamic effects of counterpulsation. IABP had no effects on heart rate. The aortic pressure and diameter waveform contour recorded in 12 patients 
Table II. Hemodynamics and aortic function data at baseline and during IABP

\begin{tabular}{|c|c|c|c|c|}
\hline Variable & Baseline & $I A B P$ & $\%$ Change & $\mathrm{P}$ \\
\hline Cycle length (ms) & $695.6 \pm 151.3$ & $699.7 \pm 158.2$ & $+0.6 \pm 0.2$ & NS \\
\hline $\mathrm{AP}_{\min }(\mathrm{mm} \mathrm{Hg})$ & $60.6 \pm 4.0$ & $51.6 \pm 3.2$ & $-14.8 \pm 1.3$ & $<.001$ \\
\hline $\mathrm{AP}_{\max }(\mathrm{mm} \mathrm{Hg})$ & $96.4 \pm 3.2$ & $87.8 \pm 2.9$ & $-9.0 \pm 0.8$ & $<.001$ \\
\hline $\mathrm{AP}_{\mathrm{a}}(\mathrm{mm} \mathrm{Hg})$ & & $109.8 \pm 2.5$ & & \\
\hline $\mathrm{AD}_{\min }(\mathrm{mm})$ & $19.6 \pm 2.1$ & $19.3 \pm 2.1$ & $-1.6 \pm 0.2$ & $<.01$ \\
\hline $\mathrm{AD}_{\max }(\mathrm{mm})$ & $20.5 \pm 2.1$ & $20.4 \pm 2.1$ & $-0.3 \pm 1.2$ & NS \\
\hline $\mathrm{AD}_{\mathrm{a}}(\mathrm{mm})$ & & $21.3 \pm 2.2$ & & \\
\hline Cardiac index $\left(\mathrm{L} \cdot \mathrm{min}^{-1} \cdot \mathrm{m}^{-2}\right)$ & $1.89 \pm 0.13$ & $2.35 \pm 0.10$ & $+24.3 \pm 3.3$ & $<.001$ \\
\hline TTI $\left(\mathrm{mm} \mathrm{Hg} \cdot \mathrm{sec} \cdot \mathrm{min}^{-1}\right)$ & $1442.8 \pm 73.0$ & $1004.1 \pm 100.6$ & $-30.5 \pm 4.7$ & $<.001$ \\
\hline Distensibility $_{1}\left(\mathrm{~cm}^{2} \cdot\right.$ dyn $\left.^{-1} \cdot 10^{-6}\right)$ & $1.89 \pm 0.09$ & $2.45 \pm 0.55$ & $+30.0 \pm 0.3$ & $<.001$ \\
\hline Distensibility $_{\mathrm{a}}\left(\mathrm{cm}^{2} \cdot \operatorname{dyn}^{-1} \cdot 10^{-6}\right)$ & & $2.72 \pm 0.20$ & & \\
\hline Slope (mm Hg/mm) & $38.42 \pm 1.02$ & $26.00 \pm 1.77$ & $-32.4 \pm 0.04$ & $<.001$ \\
\hline Intercept (mm Hg) & $-699.4 \pm 14.5$ & $-438.7 \pm 27.8$ & $+37.3 \pm 3.5$ & $<.001$ \\
\hline Stiffness constant $\left(\mathrm{mm}^{-1}\right)$ & $0.684 \pm 0.031$ & $0.599 \pm 0.032$ & $-12.5 \pm 2.9$ & $<.001$ \\
\hline Energy loss $(\mathrm{mm} \cdot \mathrm{mm} \mathrm{Hg})$ & $10.4 \pm 0.5$ & $31.9 \pm 2.6$ & $+206.6 \pm 16.3$ & $<.001$ \\
\hline Pressure augmentation index (\%) & $3.5 \pm 0.4$ & $-0.6 \pm 0.4$ & $-117.1 \pm 10.2$ & $<.001$ \\
\hline Diameter augmentation index (\%) & $10.8 \pm 0.5$ & $18.0 \pm 0.4$ & $+67.5 \pm 7.5$ & $<.001$ \\
\hline
\end{tabular}

showed characteristic changes during IABP (Fig 2). Both pressure and diameter contours showed 2 prominent peaks: the first occurred during left ventricular systole and the second during balloon inflation. Patients studied $13 \pm 5$ hours after initiation of counterpulsation showed a $9 \%$ reduction in aortic pressure at the peak of systolic and a $24 \%$ increase in cardiac index $(P<.001$; Table II). Furthermore, IABP decreased aortic pressure at the onset of systole by $15 \%(P<.001)$. Oxygen consumption studies in our patients documented a $31 \%$ decrease in TTI $(P<.001$; Table II).

Effect of counterpulsation on aortic elastic properties. During IABP, aortic distensibility during left ventricular systole (partial distensibility) increased by $30 \%$ compared with baseline $(P<.001$; Table II). Moreover total aortic distensibility calculated during the entire cardiac cycle was greater than partial distensibility $(P<.001)$.

IABP resulted in changes in the morphologic condition of the pressure-diameter relation. The ascending limb of the pressure-diameter loop was bimodal (Fig 2 ); the first peak was caused primarily by the increase in aortic pressure and diameter during left ventricular systole, and the second peak was caused by balloon inflation. The slope and the intercept of the pressurediameter relation changed $(-32 \%$ and $+37 \%$, respectively) during IABP, compared with baseline $(P<$ .001). A negative correlation was demonstrated between total aortic distensibility and the slope of the pressure-diameter relation during IABP (slope $=-4.5$ [total distensibility] $+38.3 ; \mathrm{r}=0.51 ; P<.001)$.
The aortic stiffness constant was significantly decreased by $13 \%$ during IABP compared with baseline $(P<.001$; Table II). A negative correlation was found between partial aortic distensibility and the stiffness constant both at baseline (stiffness $=-0.02$ [partial distensibility] $+0.64 ; \mathrm{r}$ $=0.51 ; P<.01)$ and during IABP (stiffness $=-0.1$ [partial distensibility $]+0.9 ; \mathrm{r}=0.56 ; P<.01)$.

Effect of counterpulsation on aortic energy loss and wave reflections. Energy loss in the aorta increased by 207\% during IABP compared with baseline $(P<.001$; Table II). The pressure augmentation index decreased significantly $(-117 \% ; P<.001)$, and the diameter augmentation index increased significantly during IABP $(+68 \% ; P<.001)$, both indicating reduced wave reflection from the arterial periphery. A negative correlation was found between pressure and diameter augmentation indices both at baseline $(r=0.94)$ and during IABP $(r=$ $0.86 ; P<.001$ for both; Fig 5).

Correlation between cardiac index, TTI, and aortic stiffness constant. There was a negative correlation between the aortic stiffness constant and the cardiac index both before and during IABP $(\mathrm{r}=0.68$ and 0.71 , respectively; $P<.001$ for both; Fig 6). Conversely, a positive correlation was demonstrated between the aortic stiffness constant and the TTI, both before and during IABP $(\mathrm{r}=0.62$ and 0.74 , respectively; $P<.001$ for both; Fig 7).

\section{Discussion}

In this study intravascular catheter measurements of the aortic diameter were combined with catheter-tip 

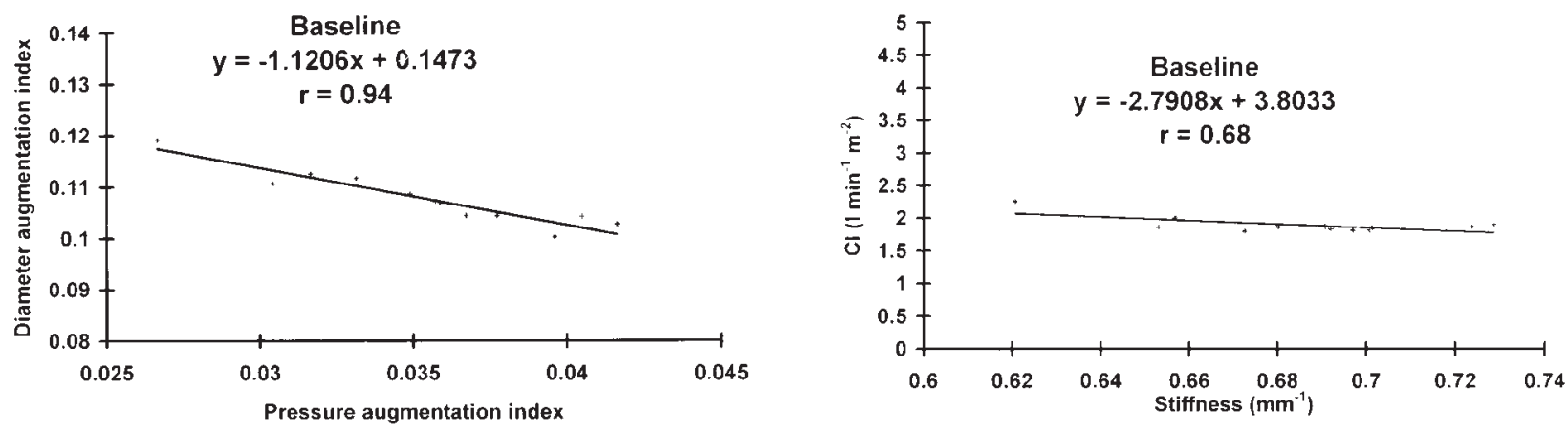

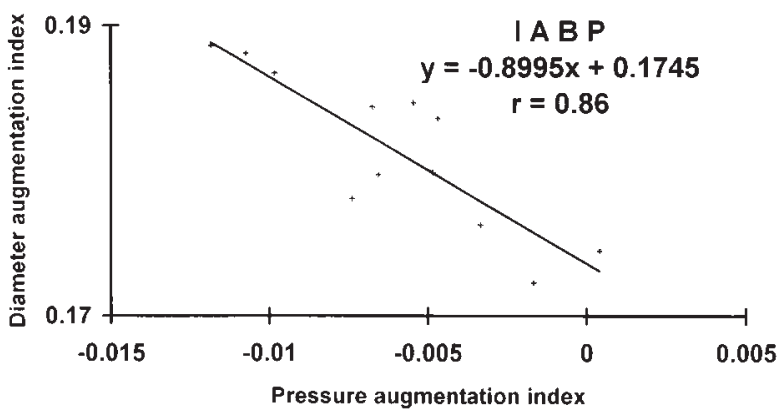

Fig 5. Correlation between the pressure augmentation index and the diameter augmentation index at baseline and during IABP.

micromanometer aortic pressure measurements to evaluate changes in aortic mechanics during IABP. Changes of the aortic pressure and diameter waveforms with IABP were characterized by a decrease of the size of the pressure systolic peak and an increase of the diameter systolic peak. A decrease of aortic stiffness was demonstrated during IABP, associated with a significant increase of the cardiac index and a significant reduction of myocardial oxygen consumption.

Effect of counterpulsation on the aortic elastic properties. IABP increased peak diastolic aortic pressure significantly; conversely, it resulted in a reduction in systolic and end-diastolic pressures. In addition the compliance of the aorta, both partial and total, was increased. Increased partial aortic compliance and decreased stiffness constant during IABP indicated an improvement of the intrinsic elastic properties of the aortic wall. Moreover, the increased total compliance and the decreased slope of the pressure-diameter relation showed further improvement of the aortic distensibility, which, either passive or active, was the result of balloon inflation. Thus the mechanical effects of the second pressure wave created by IABP on the arterial wall constituted a complementary mechanism for the hemodynamic benefit obtained with this clinically useful device.

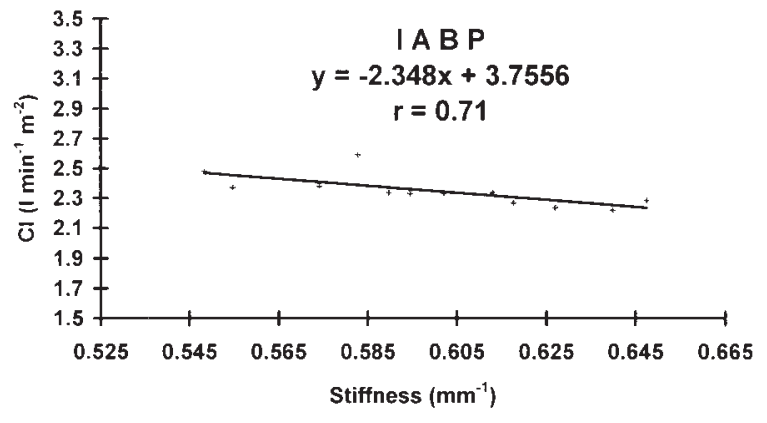

Fig 6. Inverse correlation between the aortic stiffness constant and the cardiac index $(C I)$ at baseline and during IABP.

The IABP-related decrease of the left ventricular pressure load is due primarily to the decreased aortic stiffness, which in turn decreases aortic and left ventricular pressure at its early systolic peak to the inflection point and secondarily to the reduction of wave reflection that lowers pressure from the inflection point to the late systolic peak. ${ }^{16}$

The relative role of mechanical or biochemical mechanisms to the increase of the aortic compliance remains unknown and is beyond the scope of the present investigation. However, it is noteworthy that studies have reported that nitric oxide plays an important role in flow-mediated endothelial relaxation in large arteries and that pulsatile flow itself can further enhance this effect. ${ }^{16}$ In vivo, nitric oxide production may be responsible for improvement of the aortic elastic properties and changes in blood flow pulse frequency during IABP. A number of in vitro studies performed in isolated conduit vessels have demonstrated that 1 or more endothelium-derived relaxing factors are involved in changes of pulsatility and mean flow. Furthermore, increases in pulse and mean flow increase prostacyclin release. ${ }^{21}$ Hutchenson and Griffith ${ }^{22}$ demonstrated flowdependent vasodilation in isolated perfused aortas in vitro. The possible release of the endothelial autocoids may inhibit platelet function and could also favorably 

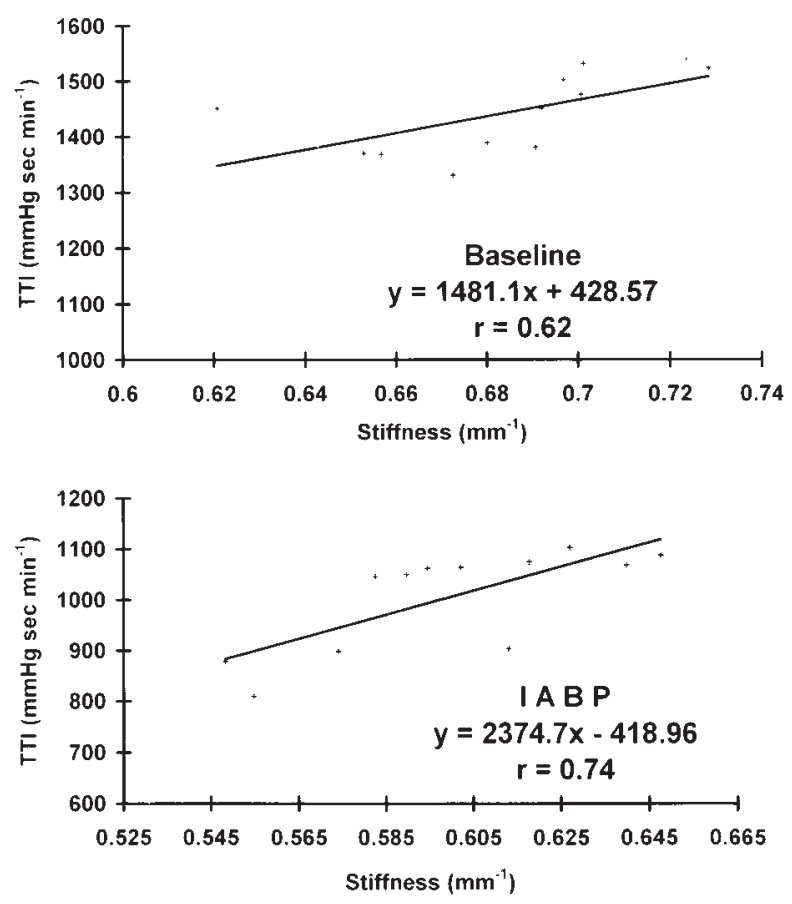

Fig 7. Correlation between the aortic stiffness constant and the tension-time index (TTI) at baseline and during IABP.

affect rethrombosis and thereby the prognosis of patients with coronary artery stenosis in whom IABP was used due to poor cardiac pumping. ${ }^{7}$

Effect of counterpulsation on cardiac and aortic energy. The most direct indicator of energy expenditure by the heart is oxygen consumption, ${ }^{6}$ which was estimated by TTI in the present study. IABP decreased cardiac energetic cost by $31 \%$. The good correlation demonstrated between TTI and aortic stiffness indicates that improved cardiac efficiency during IABP resumed from decreasing aortic stiffness. The results of our study are in agreement with the observations by Anderson and Gurbel ${ }^{7}$ and suggest that the mechanism for the improved clinical outcomes observed in patients with acute heart failure as the result of obstructive coronary artery disease after assistance by IABP is mediated by decreased myocardial oxygen demand.

We found that during IABP the aortic energy loss increased although the myocardial oxygen consumption decreased. IABP adds external energy into the aorta. Thus energy loss caused by the viscosity of the aortic wall was increased as more energy was added to the aortic wall. The decrease in left ventricular energetic cost was achieved by an increase in the aortic energetic cost.

Effect of counterpulsation on wave reflections. As IABP operates, changes are induced in the vasculature that decrease left ventricular afterload. The findings in this study agree with previously published data that show that both nonpulsatile (peripheral resistance) and pulsative (characteristic impendance and wave reflections) components of the arterial load decrease with IABP. ${ }^{6}$ Systolic unloading achieved by IABP leads to reduced myocardial oxygen demand, which seems to be the major beneficial effect of counterpulsation on the ischemic heart with severe coronary artery stenosis.

The observed increase of the aortic diameter augmentation index may be due to changes in blood flow. It seems that relations between flow and diameter are linear in the aorta. Thus the accentuated increases in flow after IABP-induced increases in diameter may be due to the additional effects of augmented systoles on conduit artery tone. Reactive dilation of the aorta may occur in response to vasodilation of the distal vascular bed.

The extent of this dilation is both flow and endothelium dependent. ${ }^{19}$ One or more endothelium-dependent relaxing factors have been implicated in local dilation of arteries in response to changes in either intraluminal flow or vessel wall shear stress. ${ }^{23}$

Furthermore, the fact that the aortic diameter augmentation index showed different but complementary changes to those of the pressure augmentation index accounts, at least in part, for the positive clinical results with IABP. Such alterations of wave reflections were largely responsible for the optimal coupling of the vasculature to the left ventricular function that occurred during IABP. These findings provide important answers to the question posed by recent studies, ${ }^{24,25}$ regarding the determinants of left ventricular function during IABP in patients with coronary artery disease.

The determination of IABP-related changes in the pulse waves by a high-fidelity diameter catheter provides important supplementary information to that obtained by use of a catheter-tip micromanometer. The use of this device promotes further investigations of the positive effects of IABP on cardiovascular function, because technically adequate simultaneous pressure and diameter signals from the same location in the ascending aorta of intact unanesthetized subjects are rendered easy to obtain.

It is concluded that IABP produces favorable effects on aortic compliance and wave reflection. Improvement of aortic elastic properties and dilation of peripheral vasculature are the major mechanisms responsible for the decreased left ventricular afterload during IABP. Reduction in pressure wave reflection causes a substantial decrease of systolic pressure in central arteries. Thus reduction in afterload and myocardial oxygen demand most likely account for the positive hemodynamic and clinical results achieved with IABP. 


\section{REFERENCES}

1. Moulopoulos SD, Topaz S, Kolff WJ. Diastolic balloon pumping (with carbon dioxide) in the aorta: a mechanical assistance to the failing circulation. Am Heart J 1962;63:669-75.

2. Hickey PR, Buckley MJ, Philbin DM. Pulsatile and nonpulsative cardiopulmonary bypass: a review of counterproductive controversy. Ann Thorac Surg 1983;36:720-37.

3. Goldberger M, Tabak SW, Prediman SK. Clinical experience with intraaortic balloon counterpulsation in 112 patients. Am Heart J 1986;111:497-502.

4. Sun Y. Modeling the dynamic interaction between left ventricle and intraaortic balloon pump. Am J Physiol 1991;261:H1300-11.

5. Williams DO, Korr KS, Gewirtz H, Most AS. The effect of intraaortic balloon counterpulsation on regional myocardial blood flow and oxygen consumption in the presence of coronary artery stenosis in patients with unstable angina. Circulation 1982;66:593-7.

6. Kim SY, Euler DE, Jacobs WR, Montoya A, Sullivan HJ, Lonchyna VA, et al. Arterial impedance in patients during intraaortic balloon counterpulsation. Ann Thorac Surg 1996;61:88894.

7. Anderson RD, Gurbel PA. The effect of intraaortic balloon counterpulsation on coronary blood flow velocity distal to coronary artery stenoses. Cardiology 1996;87:306-12.

8. Stefanadis C, Stratos C, Vlachopoulos C, Marakas S, Boudoulas $\mathrm{H}$, Kallidazaros I, et al. Pressure-diameter relation of the human aorta: a new method of determination by the application of a special ultrasonic dimension catheter. Circulation 1995;92:2210-9.

9. Stefanadis C, Boudoulas H, Toutouzas P. The aortic diameter-aortic pressure relationship in clinical practice. In: Boudoulas $\mathrm{H}$, Toutouzas P, Wooley CF, editors. Functional abnormalities of the aorta. Armond [NY]: Futura; 1996. p. 333-42.

10. Stefanadis C, Tsiamis E, Vlachopoulos C, Startos C, Toutouzas $\mathrm{K}$, Pitsavos C, et al. Unfavorable effect of smoking on the elastic properties of the human aorta. Circulation 1997;95:31-8.

11. Stratos C, Stefanadis C, Kallikazaros I, Boudoulas H, Toutouzas P. Ascending aorta distensibility abnormalities in hypertensive patients and response to nifedipine administration. Am J Med 1992;93:505-12.

12. Stefanadis C, Wooley CF, Buch CA, Kolibach AJ, Boudoulas H. Aortic distensibility abnormalities in coronary artery disease. Am J Cardiol 1987;59:1300-4.

13. Stefanadis C, Stratos C, Boudoulas H, Kourouklis C, Toutouzas P. Distensibility of the ascending aorta: comparison of invasive and non-invasive techniques in healthy men and in men with coronary artery disease. Eur Heart J 1990;11:990-6.

14. Stefanadis C, Stratos C, Boudoulas H, Vlachopoulos C, Kallikazaros I, Toutouzas P. Distensibility of the ascending aorta in coronary artery disease and changes after nifedipine administration. Chest 1994;105:1017-23.

15. Stefanadis C, Dernellis J, Vlachopoulos C, Tsioufis C, Tsiamis E, Toutouzas K, et al. Aortic function in arterial hypertension determined by pressure-diameter relation: effects of diltiazem. Circulation 1997;96:1853-8.

16. Murgo JP, Westerhof N, Giolma JP, Altobelli SA. Aortic input impedance in normal man: relationship to pressure waveforms. Circulation 1980;62:105-15.

17. Kelly R, Hayward C, Avolio A, O'Rourke M. Noninvasive determination of age-related changes in the human arterial pulse. Circulation 1989;80:1652-9.

18. Buckberg GD, Fixler DE, Archie JP, Hoffman JIE. Experimental subendocardial ischemia in dogs with normal coronary arteries. Circ Res 1972;30:67-81.

19. Canty JM, Schwartz JS. Nitric oxide mediates flow-dependent epicardial coronary vasodilation to changes in pulse frequency but not mean flow in conscious dogs. Circulation 1994;89:37584.

20. Rubanyi GM, Romero JC, Vanhoutte PM. Flow-induced release of endothelium-derived relaxing factor. Am J Physiol 1986;250: H1145-9.

21. Pohl U, Busse R, Kuon E, Bassenge E. Pulsatile perfusion stimulates the release of endothelial autocoids. J Appl Cardiol 1986; 1:215-35

22. Hutchenson IR, Griffith RM. Release of endothelium derived relaxing is mediated both by frequency and amplitude of pulsatile flow. Am J Physiol 1991;216:H257-62.

23. Bevan JA, Laher I. Pressure and flow-dependent vascular tone. FASEB J 1991;5:2267-73.

24. Kimura A, Toyota E, Lu S, Goto M, Yada T, Chiba Y, et al. Effects of intaaortic balloon pumping on septal arterial blood flow velocity waveform during severe left main coronary artery stenosis. J Am Coll Cardiol 1996;27:810-6.

25. Smalling RW, Cassidy DB, Barrett R, Lachterman B, Felli P, Amirian J. Improved regional myocardial blood flow, left ventricular unloading, and infarct salvage using an axial-flow, transvalvular left ventricular assist device: a comparison with intraaortic balloon counterpulsation and reperfusion alone in a canine infarction model. Circulation 1992;85:1152-9. 